(1)

CrossMark

\title{
Ageing lungs and very elderly COPD: anytime and anywhere
}

\author{
Joan B. Soriano ${ }^{1}$ and Samy Suissa ${ }^{2}$ \\ Affiliations: ${ }^{1}$ Instituto de Investigación Hospital Universitario de la Princesa (IISP), Universidad Autónoma de \\ Madrid, Cátedra UAM-Linde, Madrid, Spain. ${ }^{2}$ McGill University, Centre For Clinical Epidemiology, Lady Davis
} Research Institute - Jewish General Hospital, Montreal, QC, Canada.

Correspondence: Joan B. Soriano, IISP, Universidad Autónoma de Madrid, Cátedra UAM-Linde, Diego de León 62 , 28030 Madrid, Spain. E-mail: jbsoriano2đgmail.com

0

@ERSpublications

Within the current epidemiological transition, expect and find incident COPD cases anytime, anywhere http://ow.ly/UOccW

The recent European Respiratory Society/American Thoracic Society Task Force for COPD Research statement [1] requested new studies that "...evaluate the impact of age on the importance of identifying an airflow limitation (i.e. is it more important to identify asymptomatic airflow limitation in a 30-year-old than an 80-year-old?)", as well as those studies that compare outcomes among individuals diagnosed with chronic obstructive pulmonary disease (COPD) on the basis of the fixed ratio versus lower limit of normal (LLN) thresholds. In this issue of the European Respiratory Journal (ERJ), Luoto et al. [2] report the results of a study that fulfils both goals.

The world's population is ageing at a prodigious rate and it is anticipated the life span of newborns in the year 2000 in many Western countries might reach 100 years [3]. Ergo, the frequency of noncommunicable diseases will increase exponentially, and COPD, intrinsically associated with ageing and tobacco use/ exposure, is also expected to increase its burden, as many more people will live with it for longer. The recent Global Burden of Disease update estimated the number of people worldwide with COPD to be 328 million [4]. However, given the likely ecological fallacy [5], for a universally very high level of underdiagnosis [6], there are many questions that remain unanswered relating to the elderly with COPD. A research call for COPD in the elderly, similar to that for asthma in the elderly, is needed [7].

As reviewed by Luото et al. [2], there were few studies quantifying the incidence of spirometrically confirmed COPD in the literature, particularly within the elderly. As exemplified in their table 5, there is a wide heterogeneity in the published estimates of incidence, varying by method, location, age and sex. Theirs is a friendly addition, given that the LLN versus fixed ratio debate appears to be more heated than ever. Within the ERJ, it is welcome that forthcoming authors report both outcomes [8]. Thus, eventually, meta-analyses based on individual patient data will help to investigate further this question and identify the more accurate of these two approaches.

LUото et al. [2] followed for 6 years nearly a thousand individuals aged 65-100 years who had an acceptable spirometry at baseline. They participated in the longitudinal Good Aging in Skåne study, which in turn is part of the Swedish National Study on Aging and Care. Not surprisingly for an elderly population, the incidence of airflow limitation compatible with COPD was higher according to the fixed ratio definition of a forced expiratory volume in $1 \mathrm{~s} /$ (forced) vital capacity ratio $<0.7$ than with the LLN definition, as the fixed ratio underestimates airflow limitation in the young, while it is overestimated in the elderly.

This type of descriptive epidemiology research could be considered simple but even such simple studies do face complex methodological challenges. Indeed, this research has several strengths. The authors followed

Received: Nov 022015 | Accepted: Nov 062015

Conflict of interest: None declared.

Copyright @ERS 2016 
the Strengthening the Reporting of Observational Studies in Epidemiology guidance for observational studies [9], and their use of the 2012 Global Lungs Initiative reference equations [10] to assess incidence was rather novel, particularly in neatly extrapolating the lung function in those older than 95 years of age. The authors increased the response rate, which plagues such studies, by making participation flexible, by performing study visits on house calls and visits to retirement homes. Finally, they aimed to quantify selection bias from survival of the fittest by using an extrapolation of the 6-min walk distance at baseline.

However, the study naturally also has limitations, some of them already noted by the authors. The use of terbutaline as bronchodilator for spirometry was different between baseline and follow-up, with an unknown effect on the lung function measurement. However, the pre-bronchodilator data during follow-up are available and can be re-analysed to assess this effect. Moreover, machine and technician quality standards were not reported. There was also incomplete smoking information exposure, combining intermittent and continuous smokers, while no analysis of those who quit or resumed smoking over time was performed. Finally, asthma and other relevant comorbidities that could impact lung function were only dealt with in a rudimentary way.

In the end, even with the Scandinavian seal of quality for following up study participants, there was a shrinking sample size, particularly in the very elderly, again with an unknown effect of this source of potential selection bias on the estimate of incidence. This represents the greatest methodological challenge in conducting such longitudinal studies. The cohort started with 5370 people identified from the base population, which was reduced to 2025 with acceptable spirometry at baseline. Of these, however, over 1100 were excluded and could not be used to perform the follow-up lung function measurement necessary to estimate COPD incidence. In particular, the 585 who died during follow-up are the most problematic. Indeed, this exclusion is significant since it is not random, as it likely includes more of the older subjects for whom incidence of COPD was found to be especially elevated in this study. The authors suggest that such exclusions may have led to an underestimate of the true incidence "as a result of selection of the fittest". Perhaps statistical techniques that account for competing risks and control for differential exclusions could be considered in future studies to account for this depletion of susceptible subjects, thus providing some magnitude of the bias in estimating COPD incidence in this way.

To our knowledge, another study complements table 2 and figure 2 from the study by Luoto et al. [2], which report that COPD incidence measured by the fixed ratio increases with age, while incidence measured by LLN plateaus from age 60 years onwards. VAN DuRNe et al. [11] reported a decrease in COPD incidence after age 70 years. Contrary to other conditions like prostate benign hyperplasia, which are expected to affect $100 \%$ of all male centenarians, or, by the same token, fibroids in women, there are individuals who have smoked since childhood who will never develop COPD, even at an hypothetical maximum lifespan of 140 years [12]

Subsequent avenues for research can be anticipated. The primary conclusion by LUOTO et al. [2] that the LLN is better than the fixed ratio to prognosticate death at 5 years in elderly COPD individuals will satisfy the LLN community. At best, however, the results by sex and smoking lack power. We need more studies, and probably pooling of multiple cohorts, to disentangle their effect. This is a call for more descriptive epidemiology, please.

Airflow limitation and prevalence of COPD are associated with smoking, while COPD mortality and vital capacity are associated with poverty [13]. COPD incidence is probably associated with all these factors and it is also the epidemiological measure that is more challenging to determine. Estimating the incidence of nonacute conditions is difficult but estimating the incidence of chronic conditions associated with ageing and tobacco consumption is even more difficult. Within the current epidemiological transition, expect and find incident COPD cases anytime, anywhere.

\section{References}

1 Celli BR, Decramer M, Wedzicha JA, et al. An official American Thoracic Society/European Respiratory Society statement: research questions in COPD. Eur Respir J 2015; 45: 879-905.

2 Luoto JA, Elmståhl S, Wollmer P, et al. Incidence of airflow limitation in subjects 65-100 years of age. Eur Respir J 2016; 47: 461-472.

3 Christensen K, Doblhammer G, Rau R, et al. Ageing populations: the challenges ahead. Lancet 2009; 374 : $1196-1208$.

4 GBD 2013 DALYs and HALE Collaborators. Global, regional, and national disability-adjusted life years (DALYs) for 306 diseases and injuries and healthy life expectancy (HALE) for 188 countries, 1990-2013: quantifying the epidemiological transition. Lancet 2015 [In press DOI: 10.1016/S0140-6736(15)61340-X].

5 Soriano JB, Zielinski J, Price D. Screening for and early detection of chronic obstructive pulmonary disease. Lancet 2009; 374: 721-732.

6 Lamprecht B, Soriano JB, Studnicka M, et al. Determinants of underdiagnosis of COPD in national and international surveys. Chest 2015; 148: 971-985.

7 Yáñez A, Cho SH, Soriano JB, et al. Asthma in the elderly: what we know and what we have yet to know. World Allergy Organ J 2014; 7: 8. 
8 Soriano JB, Brusasco V, Dinh-Xuan AT. The European Respiratory Journal makes COPD a priority. Eur Respir J 2011; 38: 999-1001.

9 von Elm E, Altman DG, Egger M, et al. The Strengthening the Reporting of Observational Studies in Epidemiology (STROBE) statement: guidelines for reporting observational studies. Ann Intern Med 2007; 147: 573-577.

10 Quanjer PH, Stanojevic S, Cole TJ, et al. Multi-ethnic reference values for spirometry for the 3-95-yr age range: the global lung function 2012 equations. Eur Respir J 2012; 40: 1324-1343.

11 van Durme YM, Verhamme KM, Stijnen T, et al. Prevalence, incidence, and lifetime risk for the development of COPD in the elderly: the Rotterdam study. Chest 2009; 135: 368-377.

12 Ito K, Barnes PJ. COPD as a disease of accelerated lung aging. Chest 2009; 135: 173-180.

13 Burney P, Jarvis D, Perez-Padilla R. The global burden of chronic respiratory disease in adults. Int J Tuberc Lung Dis 2015; 19: 10-20. 